\title{
Prevalence of Antibiotic Resistance in Escherichia coli from the Fecal Flora of Humans in a Rural Area of Songkhla Province
}

Salisa Prasertsiriphong, M.D. ${ }^{1}$, Rattanaporn Chootong, M.D. ${ }^{1}$, Silom Jamulitrat, M.D. , Manthana Phengmak, M.Sc. ${ }^{2}$

'Division of Family Medicine, Department of Community Medicine, ${ }^{2}$ Division of Microbiology, Department of Pathology, Faculty of Medicine, Prince of Songkla University, Hat Yai, Songkhla 90110, Thailand.

Received 7 January 2019 • Revised 31 July 2019 • Accepted 8 August 2019 • Published online 8 October 2019

\section{Abstract:}

Objective: To determine the prevalence of antibiotic resistance in fecal Escherichia coli (E. coli ) isolated from humans in a rural area of Songkhla province.

Meterial and Methods: E. coli strains were isolated from the stool cultures of 75 healthy volunteers in a rural area. Resistance rates for 8 antibiotics were determined.

Results: The resistance rates for amoxicillin, doxycyclin, cotrimoxazole, gentamicin and cefazolin were 53.3, 51.3, 24.0, 5.3 and 3.3\%, respectively. No resistance to norfloxacin, ceftriaxone, and imipenem were detected.

Conclusion: The most prevalent resistant strains were found against amoxicillin. The prevalence of drug resistance in all multidrug resistant isolates were resistant to amoxicillin and doxycycline. No strains were resistant to all antibiotics in all antimicrobial categories as all the strains were found to be sensitive to ceftriazone, norfloxacin and imipenem.

Keywords: antibiotic, drug resistance, Escherichia coli, prevalence, rural area

Contact: Rattanaporn Chootong, M.D.

Division of Family Medicine, Department of Community Medicine, Faculty of Medicine, Prince of Songkla University, Hat Yai, Songkhla 90110, Thailand.

E-mail: Choratta@medicine.psu.ac.th
J Health Sci Med Res 2019;37(4):321-327 doi: 10.31584/jhsmr.201967 www.jhsmr.org 


\section{Introduction}

Antibiotic resistance is an important problem. Its incidence around the world is increased to dangerous level and now become the threat for mankind. ${ }^{1}$ In Thailand, antibiotic resistance causes 3.2 million more days of hospital stay and 38,000 deaths annually. ${ }^{2}$ Due to such a prolonged hospital stay, there is the increased risk of infection to others, resulting in massive social and economic losses. $^{2-4}$

The foremost step in the control of antibiotic resistance is the accurate identification and documentation of resistant strains possessing public health importance. ${ }^{5}$ According to the World Health Organization report ${ }^{6}$, the problem of antibiotic resistance is emerging and neglected problem in Southeast Asia region. Moreover, there are no published data on the accurate prevalence of antibiotic resistance referring to the current situation, particularly from the rural south of Thailand. Therefore, with increasing rates of antibiotic resistance, it is essential to look at the prevalence of drug resistance in a specific geographical region to simplify clinicians in choosing the proper antimicrobial therapy, to design and manage infection control.

Moreover, currently, there is a lack of settled global standards for antibiotic resistance surveillance ${ }^{6}$ and few studies have examined the prevalence of antibiotic resistance in Escherichia coli (E. coli) from rural area of Thailand. Therefore, this cross-sectional descriptive study reports the prevalence of antibiotic resistance in E. coli from human in rural area for the benefits of public health of Thailand.

\section{Materiall and Methods}

\section{Study setting}

The study was performed at Hin Kliang village (village no. 6), Thakham sub-district, Hat Yai district, Songkhla province, which is a farming village in the southern part of Thailand. It has a low population density. The study protocol was approved by the ethics committee of Faculty of Medicine, Prince of Songkla University, Songkhla province, Thailand (EC no. 60-466-09-4).

\section{Study population}

The volunteers that participated in this study were 18 years old and above who were free from recent antibiotic exposure for at least one month. The exclusion criterion was the people with diarrhea used during 1 month before participation. Data collection took place between 1 March 2018 and 30 April 2018. Volunteers were informed consent and provided the personal information through the questionnaires (general characteristics, history of illness and treatment).

\section{Determination of sample size}

Finite population proportion was used for calculating the suitable sample size ${ }^{7}$ as followed.

Where $\mathrm{N}$ was population size $=453$, $n$ was sample size with finite population correction, $P$ was expected proportion $=0.67$, $d$ was precision $=0.1$ and $\alpha$ was 0.05 .

The sampling was random by 2 stage random sampling. The 75 families were taken by systematic random sampling in computer program. The people who are 18 years old and above for each family were random by simple random sampling by computer. The sampling was excluded the person who used antibiotics or had diarrhea in one month. If sampling is not matched with criteria, they were repeated random.

\section{Sample collection}

A total of 75 samples were obtained by rectal swab procedure. Briefly, sterile cotton swab was applied into the anal canal approximately $3 \mathrm{~cm}$ deep, kept and transported by Stuart's transport media (Oxiod, Hamshire, UK) to the laboratory within 2 hours.

\section{Identification of $E$. coli}

In order to isolate $E$. coli from the samples, samples from transport media were cultured on MacConkey agar. After plate incubation at $35 \pm 2{ }^{\circ} \mathrm{C}$ for $16-24$ hours, $3-4$ surrogated colonies were chosen for $E$. coli identification by biochemical test. 


\section{Antimicrobial susceptibility assay}

Antimicrobial susceptibility of $E$. coli was performed using an agar disk diffusion method (CLSI, 2014) ${ }^{7}$ using 8 antimicrobial agents, namely, amoxicillin $(10 \mu \mathrm{g})$, cefazolin $(30 \mu \mathrm{g})$, ceftriaxone $(30 \mu \mathrm{g})$, cotrimoxazole $(1.25 / 23.75 \mu \mathrm{g})$, doxycycline $(30 \mu \mathrm{g})$, gentamicin $(30 \mu \mathrm{g})$, imipenem (10 $\mu \mathrm{g})$, and norfloxacin $(10 \mu \mathrm{g})$ (Oxiod, Hamshire, UK). E. coli ATCC 25922 was used as a control.

\section{Statistical analysis}

Descriptive statistics were used for describing characteristics and prevalence of antibiotic resistance. For the descriptive data, mean value was used to represent the continuous variables, and the proportion value was used to describe the categorical data. The distribution of continuous variables for normality was tested. The data were computerized by statistics and data $\left(\right.$ STATA $\left.^{\circledR}\right)$ version 10.3.

\section{Results}

\section{Demographic data of participants}

A total, 75 healthy volunteers (20 for male and 55 for female) participated in this study. The mean age was 50.7 years old. The educational level of the most volunteers was primary school graduates $(42.7 \%)$ and most of them were Buddhist. The majority were farmers $(61.3 \%)$ who earned less than or equal to 5,000 Baht per month (65.3\%). Moreover, the main health insurance plan was found to be the Universal Health Care (88.0\%).

\section{History of illness and treatment of participants}

Most volunteers had no antibiotic exposure in the previous 6 months (89.3\%), and all of them had no history of implanted urine catheter in the previous year (100.0\%). Most volunteers had no history of hospitalization in the previous year (94.7\%), nor did they have any prior urinary tract infection within the previous year (90.7\%).

\section{Bacterial isolate and the prevalence of antibiotic} resistance

A total of 150 E. coli isolates were obtained from the fecal samples of 75 healthy volunteers in a rural area. Their antibiotic resistant abilities were analyzed by agar disc diffusion assay using 8 antibiotic agents.

The results demonstrated that $E$. coli strains were resistant to amoxicillin, doxycycline, cotrimoxazole, gentamicin and cefazolin were 53.3, 51.3, 24.0, 5.3 and 3.3, respectively. Nevertheless, the resistance to ceftriaxone, norfloxacin and imipenem were not found.

\section{Prevalence of multidrug resistant (MDR) bacteria}

Our data showed 4 patterns of MDR. The prevalence of drug resistance in all $(100.0 \%)$ of the isolates of MDR were resistant to amoxicillin and doxycycline. No strains were resistant to all the antibiotics in all the antimicrobial categories as all the strains were found to be sensitive to ceftriazone, norfloxacin and imipenem (100.0\%). The percentage of MDR to each antibiotic is shown in Table 1.

Table 1 Prevalence of multidrug resistance to each antibiotic

\begin{tabular}{|l|l|l|l|l|l|l|}
\hline \multicolumn{4}{|c|}{ Antimicrobials } & \multicolumn{2}{c|}{ Non-susceptible } \\
\hline AMP & DOX & SXT & GEN & CFZ & Number & $\%$ \\
\hline & & & & & 5 & 3.3 \\
\hline & & & & & 2 & 1.3 \\
\hline & & & & & 25 & 16.7 \\
\hline & & & & & 3 & 2.0 \\
\hline
\end{tabular}

The isolate is non-susceptible to all agents listed in category The isolate is susceptible to all agents listed in category

$\mathrm{AMP}=$ ampicillin, DOX=doxycycline, $\mathrm{SXT}=$ trimethop rim/sulfamethoxazole, GEN=gentamicin, CFZ=cefazolin

The MDR in Enterobacteriaceae was defined as resistant to three or more antimicrobial classes. 


\section{Discussion}

Antibiotic resistance continues to be a major global challenge in the management of bacterial infection. The trouble behind antibiotic resistance is highly marked in undeveloped and developing countries, including Thailand. ${ }^{29}$ Factors responsible for an increase in the rates of antibiotic resistance include overuse and misuse of antibiotics and inadequate surveillance systems due to a lack of stringent antibiotic policies and guidelines from the country, leading to the inappropriate prescription of antibiotics. ${ }^{30}$ Another leading cause of the emergence of antibiotic resistance in common pathogens is the unnecessary use of antibiotics without a prescription for the treatment of common bacterial infection. ${ }^{10}$

There is little information regarding the antibiotic resistance of fecal $E$. coli isolates from rural areas in Thailand. Therefore, in this study, the prevalence of antibiotic resistance in fecal $E$. coli isolates from healthy volunteers in a rural area was examined.

This study wanted to determine the prevalence of antibiotic resistance in fecal $E$. coli isolated from humans in a rural area. The study population included the general population who were 18 years old and above from Hin Kliang village (village no. 6), Thakham sub-district, Hat Yai district, Songkhla province and free from recent antibiotic exposure or diarrhea for at least one month. The specimens were collected from $1^{\text {st }}$ March 2018 to $30^{\text {th }}$ April 2018. The resistance rates for eight antibiotics were determined from $E$. coli stains isolated.

The results of antibiotic resistance in the current study exhibits that the most prevalent of resistant were found against amoxicillin (53.3\%), which is in the penicillin group of antibiotics. In comparison with a previous study in the rural areas in Thailand, in 1988 found that the most prevalent antibiotic was tetracycline (25.8\%). ${ }^{9}$ The reason may be because tetracycline was developed before 1950; it was an extensively used drug and well known in former times but amoxicillin was developed after the 1970s. If compared with the same group of antibiotics as penicillin, this study found the prevalence of amoxicillin resistance (53.3\%) was higher than in a previous study ${ }^{9}$, in which the prevalence of ampicillin resistance (16.7\%) while in the current situation the resistance rate was more than 3 times higher, but lower than the prevalence of amoxicillin resistance in Songklanagarind Hospital (74.8\%).The reason may be because the unnecessary use of antibiotics without prescription is higher than previously; and in the hospital, the extensive use of antibiotics and close contact amongst patients makes for a productive environment for the spread of antimicrobial resistance plasmid.

When compared with the rural areas of other countries such as Korea, Philippines, India, Venezuela, Mexico, Peru, Kenya, Ghana Zimbabwe and Etiopia ${ }^{11-27}$, the maximum levels of resistance were found in amoxicillin and oxytetracycline. The highest maximum levels of resistance in urban areas were found in amoxicillin, oxytetracycline, and trimethoprim. The association of resistance to these three antibiotics is very likely due to the gene encoding resistance to these antimicrobials that are located on the same plasmid. ${ }^{28}$

Resistance was lower in urban areas in most previous studies, such as Nys, 2004 in urban Mexico ${ }^{11}$ the prevalence was $78.8 \%$ and the urban Philippines ${ }^{11}$ was $82.9 \%$. The reason may be because of the ability to access the antibiotics rate in this era; and, in urban areas, it was higher than in rural areas.

In a previous study ${ }^{9}$, no resistance to aminoglycoside was detected The author declared, 'because routes of drug administration were injection', but in this study, the prevalence of gentamicin resistance was $5.3 \%$. These data indicated that although the routes of drug administration were injection, the prevalence was higher than in the previous study. ${ }^{9}$ The reason may be not only the unnecessary use of antibiotics without prescription, but also overuse in the hospital.

No resistance to carbapenem was detected, which is different from Ramalingam, $2016^{16}$ in India was $21.4 \%$. It may be because this study collected stool 
samples from patients in a hospital where the extensive use of antibiotics and close contact amongst patients made for a productive environment for the spread of antimicrobial resistance plasmid.

The maximum levels of resistance were found in ampicillin $(74.8 \%)$, which is in the penicillin group of antibiotics. ${ }^{31}$ In comparison with this study, the maximum levels of resistance were found in amoxicillin (53.3\%), which is in the same group of antibiotics.

Our data showed that the prevalence of drug resistance in all (100.0\%) of the isolates of MDR were resistant to amoxicillin and doxycycline. The most common pattern of MDR was amoxicillin, doxycycline and cotrimoxazole $(16.7 \%)$. The reason may be because those drugs have been developed for a long time and are easy to access from drugstores.

The results of this study could assist in increasing the evidence available in order to make sensible judgments on antibiotic therapy both at the level of the practicing clinician and at the level of the authorities responsible for developing stringent antibiotic policy and guidelines. Moreover, our findings indicate the higher magnitude of $E$. coli resistance. This may imply the need for intervention in prescribing and using antibacterial agents against $E$. coli infections. Interventional strategies may include creating public awareness, maintaining hand hygiene, applying infection prevention protocols, and maintaining environmental sanitation, all of which are encouraged for preventing infection. In addition to these, the identification and documentation of resistant strains possessing public health significance is the foremost step in the control of antibiotic resistance. Promoting health education, maintaining continuous professional education, and advocating rational prescribing habits are evidently effective in the minimization of the unwanted use of antibiotics, which in turn decreases the selective pressure of resistant strains. We used documentation from this study to create community projects to improve awareness, under- standing of antibiotic resistance, and maintaining behaviors for the rational use of antibiotics for the people of Hin Kliang village (village no. 6), Thakham sub-district, Hat Yai district, Songkhla province, who cooperated with village health volunteers.

Furthermore, this study can reference the current situation of antibiotic resistance in rural Thailand, and is useful in comparison with the prevalence of antibiotic resistance in the future.

The variations in the study might be due to differences in study locations and procedures and antimicrobial utilization. This is a cross-sectional descriptive study and we used questionnaires recorded by volunteers; thus, the information that we received from the questionnaires may have had information bias, but we tried to decrease the bias by explaining the information sheet about the protocols. This study may also have had recall bias, because part 2 of the questionnaire asked about their history of illness and treatment, and some volunteers may have forgotten the true information. However, we prevented this bias by using the reference period to remind them about the situation. And, the last bias that may have occurred in this study was sampling bias, but we tried to eliminate this bias by using stage 2 random sampling, both systematic random sampling and simple random sampling.

This study was limited by its small sample size due to limited time and finances. The factors of antibiotic resistance remain unknown, and therefore require further investigation.

\section{Conclusion}

This study provides useful information on the prevalence of antibiotic resistance. Furthermore, it increases the awareness of resistance issues. This study may also help to manage the evolution of antibiotic resistance in the future. Adopting safety protocols and implementing proper antibiotic prescription policies could be potential 
interventional strategies and suggest further evidence of the need for commitment to ensuring antibiotics are used in as rational a manner as possible.

This study suggests further investigation into the problem encompassing broader adjoining areas of Southeast Asia that share geography and have related living practices to design a broad policy for the containment of the problem in Southeast Asia.

\section{Acknowledgement}

We would like to express our gratitude to the Faculty of Medicine, Prince of Songkla University for both supporting and funding our study. We also appreciate the coordinators from the Division of Microbiology, Songklanagarind Hospital for their kind assistance.

In addition, we thank all the staff of the Family Medicine and Preventive Medicine, Prince of Songkla University for their help.

\section{Conflict of Interest}

We have no competing interests to declare, and confirm that the data we collected and used for the study is original.

\section{References}

1. Ventola CL. The antibiotic resistance crisis part 1: causes and threats. J Clin Pharm Ther 2015;40:277-83.

2. Phumart P, Phodha T, Thamlikitkul V, Riewpaiboon A, Prakongsai $\mathrm{P}$, Limwattananon S. Health and economic impacts of antimicrobial resistance in Thailand. J Health Systems Res 2012; 6:352-60.

3. Cosgrove SE. The relationship between antimicrobial resistance and patient outcomes: mortality, length of hospital stay, and health care costs. Clin Infect Dis 2006;42(Suppl 2):S82-9.

4. Lautenbach E, Patel JB, Bilker WB, Edelstein PH, Fishman NO. Extended-spectrum beta-lactamase-producing Escherichia coli and Klebsiella pneumoniae: risk factors for infection and impact of resistance on outcomes. Clin Infect Dis 2001; 32:1162-71.
5. Prestinaci F, Pezzotti P, Pantosti A. Antimicrocial resistance: a global multifaceted phenomenon. Pathog Glob Health 2015; 109:309-18.

6. World Health Organization. on surveillance: antibiotic resistance [monograph on the Internet]. Geneva: WHO; 2014 [cited 2017 Oct 26]. Available from: http://Geneva,who.int/iris/bitstream/ 10665/112642/1/9789241564748_eng.pdf

7. Daniel WW. Biostatistics: a foundation for analysis in the health Sciences. $9^{\text {th }}$ ed. New York: John Wiley \& Sons 2019; 189-214.

8. Jean B, Franklin R, Jeff A, Patricia A, George M, Dwight J. Performance standards for antimicrobial susceptibility testing; twenty-fourth informational supplement. Clin Lab Standards Ins 2014;24:62-67.

9. Jamulitrat S, Promplook S, Varindsathien P. Antibiotic resistance pattern of Escherichia coli isolated from fecal flora of human in rural area, J infect Dis Antimicrob Agents 1998;5:6-8.

10. Antibiotic resistance: drug resistance, antimicrobial resistance [monograph on the Internet]. Florida: Infectious Disease Health Center; 2016 [cited 2016 Jul 10]. Available from: https://www. medicinenet.com/antibiotic_resistance/article.htm\#the_history_ of_antimicrobial_drug_resistance

11. Nys S, Okeke I, Kariuki S, Dinant G, Driessen C, Stobberingh E. Antibiotic resistance of faecal Escherichia coli from healthy volunteers from eight developing countries. J Antimicrob Chem 2004;54:952-5.

12. Wuttananupan K. Antibiotic resistance pattern and resistance genes of Escherichia coli isolates from human stool samples [Dissertation]. Songkhla: Prince of Songkla University; 2006.

13. Cho S, Lim Y, Park M, Kim S, Kang Y. Prevalence of antibiotic resistance in Escherichia coli fecal isolates from healthy persons and patients with diarrhea. Public Health Res Perspect 2011;2:41-5.

14. Shin $\mathrm{H}$, Cho $\mathrm{S}$. Prevalence of antimicrobial resistance in Escherichia coli strains isolated from fishery workers, Osong Public Health Res Perspect 2013;4:72-5.

15. Adugna A, Kibret M, Abera B, Nibret E, Adal M, Antibiogram of $E$. coli serotypes isolated from children aged under five with acute diarrhea in Bahir Dar town. Afr Health Sci 2015;15:65664.

16. Sekar R, Mythreyee M, Srivani S, Amudhana M. Prevalence of antimicrobial resistance Klebsiella spp. in rural South India. J Glob Antimicrob Res 2016;5:80-5. 
17. Desta K, Woldeamanuel Y, Azazh A. High gastrointestinal colonization rate with extended-spectrum $\beta$-lactamase producing Enterobacteriaceae in hospitalized patients: emergence of carbapenemase-producing $\mathrm{K}$. pneumoniae in Ethiopia. PLoS ONE 2016;11:54-64.

18. Beyene G, Tsegaye W. Bacterial uropathogens in urinary tract infection and antibiotic susceptibility pattern in Jimma University specialized hospital, southwest Ethiopia. Ethiop J Health Sci 2011;21:61-5.

19. Demilie T, Beyene G, Melaku S, Tsegaye W. Urinary bacteria profile and antibiotic susceptibility pattern among pregnant women in north west Ethiopia. Ethiop J Health Sci 2012;22: 121-8.

20. Abejew A, Denboba A, Mekonnen A. Prevalence and antibiotic resistance pattern of urinary tract bacterial infections in Dessiearea, Northeast Ethiopia. BMC Res Notes 2014;7: 687-8.

21. Tiruneh M, Yifru S, Gizachew M. Changing trends in prevalence and antibiotics resistance of uropathogens in patients attending the Gondar University Hospital, Northwest Ethiopia. Int J Bacteriol 2014;14:1-7.

22. Eshetie S, Tarekegn F, Kumera G, Mekonnen F. Multidrug resistant Escherichia coli strains isolated from urine sample, University of Gondar Hospital, Northwest Ethiopia. J Coastal Life Med 2016;4:140-2.

23. Derbie A, Hailu D, Mekonnen D, Abera B, Yitayew G. Antibiogram profile of uropathogens isolated at Bahir Dar regional health research laboratory centre, northwest Ethiopia. Pan Afr Med J 2017;26:134

24. Dereje M, Woldeamanuel Y, Asrat D, Ayenachew F. Urinary tract infection among fistula patients admitted at Hamlin fistula hospital, Addis Ababa, Ethiopia. BMC Infect Dis 2017; $17: 150$.

25. Wondimeneh Y, Muluye D, Alemu A. Urinary tract infection among obstetric fistula patients at Gondar University Hospital, Northwest Ethiopia. BMC Womens Health 2014;14:12.

26. Wasihun A, Wlekidan L, Gebremariam S. Bacteriological profile and antimicrobial susceptibility patterns of blood culture isolates among febrile patients in Mekelle hospital, Northern Ethiopia. Springerplus 2015;4:1.

27. Mulu W, Abera B, Yimer M, Hailu T, Ayele H, Abate D. Bacterial agents and antibiotic resistance profiles of infections from different sites that occurred among patients at Debre Markos Referral Hospital, Ethiopia: a cross-sectional study. BMC Res Notes 2017;10:254.

28. Richard J, Yitzhak T. Antibiotics and bacterial resistance in the $21^{\text {st }}$ Century. Perspect Med Chem 2014;6:25-64.

29. Hart A, Kariuki S. Antimicrobial resistance in developing countries. BMJ 1998;317:647-50.

30. Zamen S, Hussain M, Nye R, Mehta V, Mamun K, Hossain N. A review on antibiotic resistance: Alarm bells are ringing. Cureus 2017;9:1-9.

31. Songklanagarind Hospital. Nursing Services Division. Statistics of wards in 2018. Songkhla: The Hospital; 2018. 